\title{
Evaluation of the stability of cucurbit[8] uril-based ternary host-guest complexation in physiological environment and the fabrication of a supramolecular theranostic nanomedicine
}

\author{
Han Wu ${ }^{1}$, Zuobing Chen ${ }^{2^{*}}$, Shaolong Qi ${ }^{1}$, Bing Bai ${ }^{2}$, Jiajun $\mathrm{Ye}^{3}$, Dan Wu ${ }^{4}$, Jie Shen ${ }^{5}$, Fei Kang ${ }^{3^{*}}$ and \\ Guocan $\mathrm{Yu}^{1 *}$ (D)
}

\begin{abstract}
Background: Supramolecular theranostics have exhibited promising potentials in disease diagnosis and therapy by taking advantages of the dynamic and reversible nature of non-covalent interactions. It is extremely important to figure out the stability of the driving forces in physiological environment for the preparation of theranostic systems.

Methods: The host-guest complexation between cucurbit[8]uril (CB[8]), 4,4'-bipyridinium, and napththyl guest was fully studied using various characterizations, including nuclear magnetic resonance spectroscopy, ultraviolet-visible (UV-Vis) spectroscopy, isothermal titration calorimetry (ITC). The association constants of this ternary complex were determined using isothermal titration calorimetry. The stability of the non-covalent interactions and self-assemblies form from this molecular recognition was confirmed by UV-vis spectroscopy and dynamic light scattering (DLS). A supramolecular nanomedicine was constructed on the basis of this 1:1:1 ternary recognition, and its in vitro and in vivo anticancer efficacy were thoroughly evaluated. Positron emission tomography (PET) imaging was used to monitor the delivery and biodistribution of the supramolecular nanomedicine.

Results: Various experiments confirmed that the ternary complexation between 4,4'-bipyridinium, and napththyl derivative and $\mathrm{CB}[8]$ was stable in physiological environment, including phosphate buffered solution and cell culture medium. Supramolecular nanomedicine (SNM@DOX) encapsulating a neutral anticancer drug (doxrubincin, DOX) was prepared based on this molecular recognition that linked the hydrophobic poly( $\varepsilon$-caprolactone) chain and hydrophilic polyethylene glycol segment. The non-covalent interactions guaranteed the stability of SNM@DOX during
\end{abstract}

\footnotetext{
*Correspondence: czb1971@zju.edu.cn; fmmukf@qq.com; guocanyu@mail.

tsinghua.edu.cn

${ }^{1}$ Key Laboratory of Organic Optoelectronics and Molecular Engineering,

Department of Chemistry, Tsinghua University, Beijing 100084, People's

Republic of China

2 Department of Rehabilitation Medicine, The First Affiliated Hospital,

College of Medicine, Zhejiang University, Hangzhou 310003, People's

Republic of China

${ }^{3}$ Department of Nuclear Medicine, Xijing Hospital, Fourth Military

Medical University, Xi'an 400030, People's Republic of China

Full list of author information is available at the end of the article
}

(c) The Author(s) 2021. Open Access This article is licensed under a Creative Commons Attribution 4.0 International License, which permits use, sharing, adaptation, distribution and reproduction in any medium or format, as long as you give appropriate credit to the original author(s) and the source, provide a link to the Creative Commons licence, and indicate if changes were made. The images or other third party material in this article are included in the article's Creative Commons licence, unless indicated otherwise in a credit line to the material. If material is not included in the article's Creative Commons licence and your intended use is not permitted by statutory regulation or exceeds the permitted use, you will need to obtain permission directly from the copyright holder. To view a copy of this licence, visit http://creativecommons.org/licenses/by/4.0/. The Creative Commons Public Domain Dedication waiver (http://creativeco mmons.org/publicdomain/zero/1.0/) applies to the data made available in this article, unless otherwise stated in a credit line to the data. 
blood circulation and promoted its tumor accumulation by taking advantage of the enhanced permeability and retention effect, thus greatly improving the anti-tumor efficacy as compared with the free drug.

Conclusion: Arising from the host-enhanced charge-transfer interactions, the CB[8]-based ternary recognition was stable enough in physiological environment, which was suitable for the fabrication of supramolecular nanotheranostics showing promising potentials in precise cancer diagnosis and therapy.

Keywords: Supramolecular chemistry, Host-guest molecular recognition, Nanomedicine, Drug delivery, Chemotherapy

\section{Introduction}

Supramolecular theranostics combining diagnostic and therapeutic functions into one platform constructed from non-covalent interactions have attracted extensive attentions over the past years owing to their unparalleled advantages [1-4]. Imaging probes, therapeutic agents and targeting ligands can be integrated into the theranostic system through self-assembly on the basis of supramolecular chemistry, greatly avoiding tedious organic synthesis [5-7]. Additionally, the dynamic and reversible nature of non-covalent interactions endow the supramolecular theranostics with brilliant stimuli-responsiveness, activating the loaded drugs in sites of action triggered by the specific tumor microenvironment, possibly optimizing the theranostic performance and decreasing undesirable side effects [8-10]. Various non-covalent interactions have been employed to construct supramolecular theranostics, such as H-bonds, van der Waals forces, metal coordinations, electrostatic interactions and host-guest complexations [11, 12]. Attributing to their abundant responsivenesses to endogenous or exogenous stimuli, host-guest systems have gained numerous attentions in the development of supramolecular theranostics [13-17]. Macrocyclic hosts, like cyclodextrins, calixarenes, cucurbiturils, and pillararenes are widely utilized for the treatment of cancer and other diseases, which have achieved impressive outcomes [18-22]. Due to the differences in topological structures and substituents, the binding behaviors and affinities of these hosts with guest molecules are always distinct, which are extremely important for the fabrication of supramolecular theranostics.

Different from traditionally covalent bonds, non-covalent interactions show relatively weak affinities, which can be interfered by the external triggers in physiological environment. For example, $\mathrm{H}$-bonds are remarkably impaired in aqueous solution by water molecules that act as $\mathrm{H}$-bonds donors and acceptors. Electrostatic interactions and metal coordinations can be extremely weakened in the presence of cations, such as iron, copper, zinc et $c$, which largely exist in the body fluids. In the case of host-guest inclusion complexes, their interactions are sensitive to the salts, peptides, and proteins, which can also work as competitive molecules to interact with the hosts or guests. Considering the rigid structures and high association constants towards guests, cucurbiturils have been widely chosen for the construction of supramolecular polymers, sensors and drug delivery systems [23-33]. Especially for cucurbit[8]uril ( $\mathrm{CB}[8])$, its large cavity size $\left(479 \AA^{3}\right)$ allows the formation of heteroternary complexes in the hollow cavity with a well-defined face-to-face $\pi-\pi$ stacking geometry of the electron-rich donor and electron-deficient acceptor [34, 35]. This recognition motif is a practical donor-acceptor mix-and-match approach, the acceptor and donor molecules separately penetrates into the cavity as the first and second guest $[36,37]$. The ability to form 1:1:1 ternary host-guest complexes distinguishes $\mathrm{CB}[8]$ from other macrocycles in the development of supramolecular nanomedicines, in which $\mathrm{CB}[8]$ acts as non-covalent linker to connect functional building blocks. Although this molecular recognition shows bright future in biomedical application, its stability in physiological environment is rarely evaluated.

Herein, we fully investigate the ternary host-guest complexation between $\mathrm{CB}[8], 4,44^{\prime}$-bipyridinium, and napththyl derivative, and determine the association constants in PBS and cell culture medium. The strong binding affinities ensure the heteroternary complexation in these physiological solutions, guaranteeing the stability of supramolecular delivery systems prepared from this host-guest recognition. Indeed, a supramolecular deblock copolymer is prepared using 4,4'-bipyridiniummodified poly( $\varepsilon$-caprolactone) (PCL-MV), 6-methoxy2-naphthol-conjugated methoxy poly(ethylene glycol) (Nap-PEG) and $\mathrm{CB}[8]$ as building blocks (Scheme 1), which self-assembles into supramolecular nanoparticles (SNPs) in aqueous solution with the ability to encapsulate a hydrophobic anticancer drug DOX to afford a supramolecular nanomedicine (SNM@DOX). Benefiting from the strong binding affinities, SNM@DOX maintains intact in physiological environment, a pivotal role in the prevention of premature drug release. The supramolecular nanoformulation is readily labelled by a radioactive tracer $\left({ }^{89} \mathrm{Zr}\right)$ through host-guest chemistry, monitoring its delivery in real time by PET imaging. Compared with free drug (doxorubicin hydrochloride, DOX. $\mathrm{HCl}$ ), the circulation time and tumor accumulation are greatly 


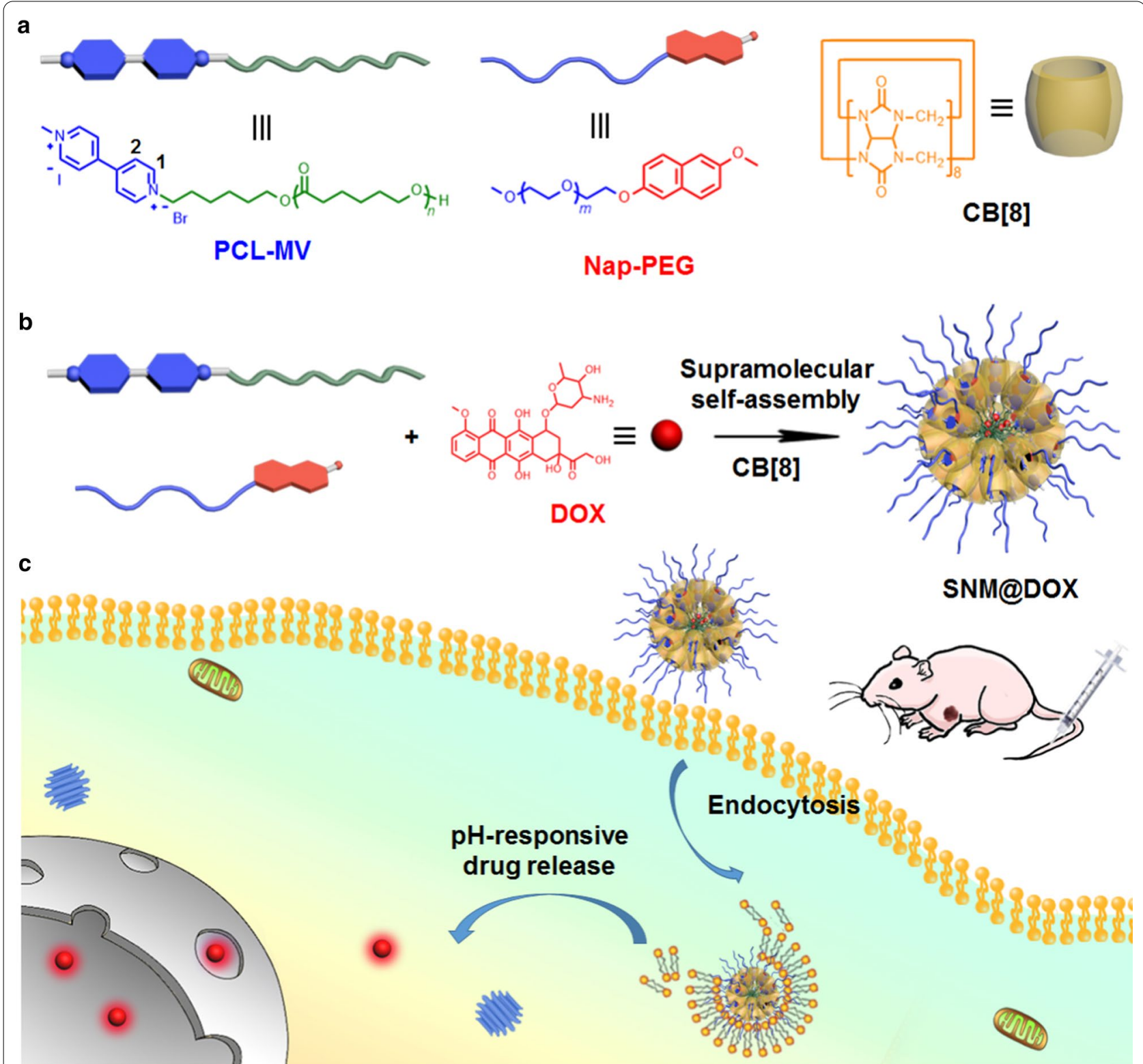

Scheme 1 a The chemical structures of CB[8], Nap-PEG and PCL-MV. b The preparation of supramolecular nanomedicine SNM@DOX.c Schematic illustration of the drug delivery and cellular internalization of SNM@DOX.

increased mainly attributing to the enhanced permeability and retention (EPR) effect. In vivo experiments demonstrate the excellent anti-tumor performance and low systemic toxicity of SNM@DOX.

\section{Results}

\section{Characterizations of host-guest complexation}

Proton nuclear magnetic resonance $\left({ }^{1} \mathrm{H}\right.$ NMR) spectroscopy was firstly utilized to characterize the host-guest complexation using methylviologen (MV), and Nap-PEG as model guests (Fig. 1a-e). As shown in the ${ }^{1} \mathrm{H}$ NMR spectra collected in PBS using $\mathrm{D}_{2} \mathrm{O}$ as a solvent, upfield shift changes were monitored for the signals related to the protons $\left(\mathrm{H}_{1}\right.$ and $\left.\mathrm{H}_{2}\right)$ of $\mathrm{MV}$ upon addition of $\mathrm{CB}[8]$, indicating that the cationic guest deeply threaded into the cavity (Fig. 1d). The driving forces for the inclusion complexation benefited from the hydrophobic cavity and dipolar nature of the carbonyl-fringed portals of $\mathrm{CB}[8]$, which made the host highly attractive for dicationic guests where the cations were separated by a hydrophobic region through hydrophobic interactions and ion-dipole effect [35]. Notably, negligible changes in 


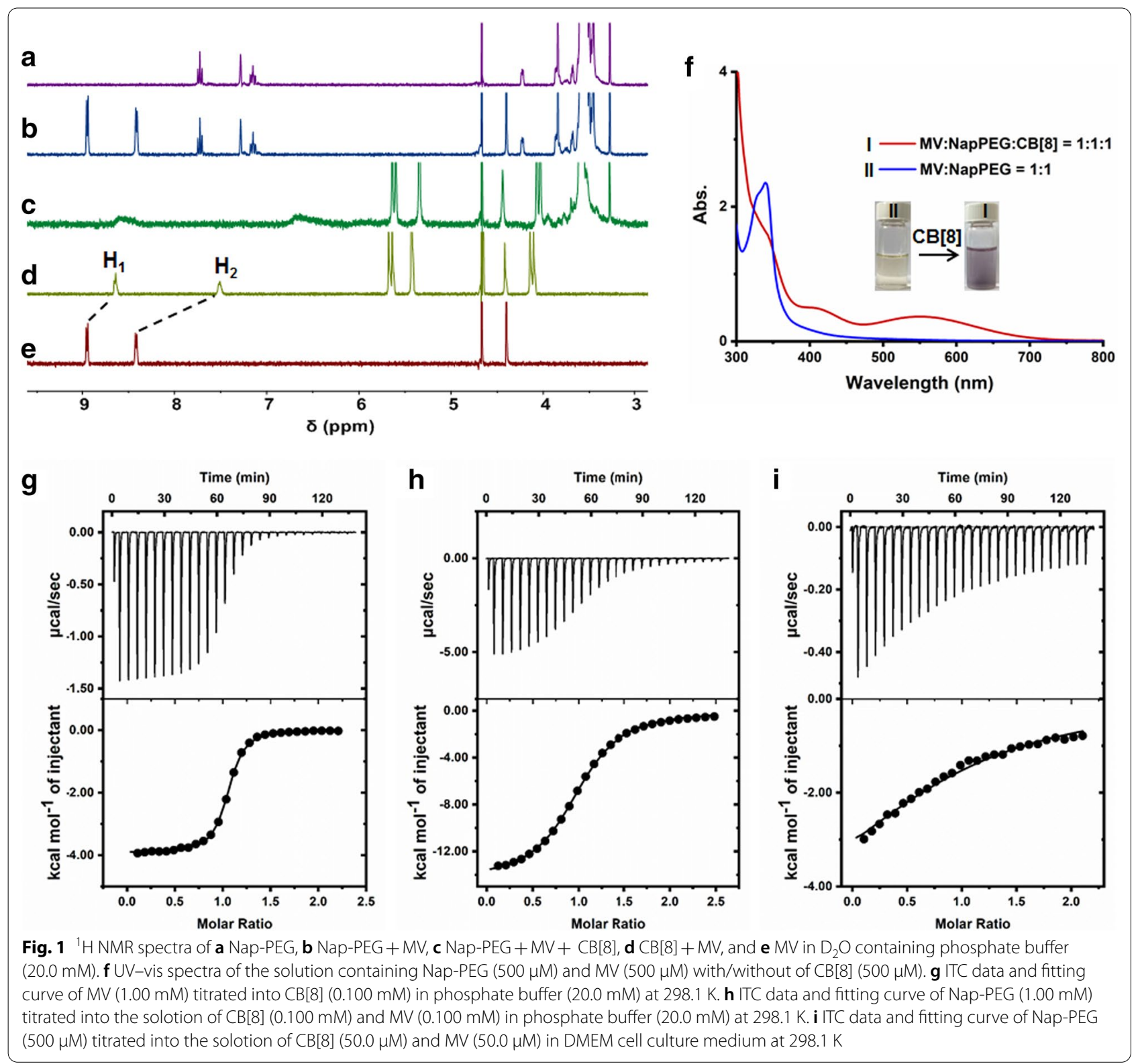

chemical shift were detected for peaks of Nap-PEG in the presence of MV (Fig. 1b), confirming negligible interactions between these two building blocks without $\mathrm{CB}[8]$. Interestingly, obvious changes were observed upon addition of $\mathrm{CB}[8]$ into the solution containing Nap-PEG and MV (Fig. 1c). The peaks of the aromatic protons on NapPEG and MV disappeared due to the broaden effect, and the signal related to the protons of $\mathrm{CB}[8]$ shifted upfield, a convincing evidence for the formation of ternary host-guest complex. Host-enhanced charge-transfer (CT) interactions induced the formation of a 1:1:1 complex, because $\mathrm{CB}[8]$ possessed a large cavity that is able to encapsulate Nap-PEG and MV simultaneously, where
MV acted as the electron acceptor and Nap-PEG acted as the electron donor.

Further evidence for the formation of CT interactions in the cavity of $\mathrm{CB}[8]$ came from UV-vis absorption spectroscopy (Fig. 1f). In sharp comparison with the mixture of Nap-PEG and MV in PBS, a broad absorption was measured ranging from 400 to $700 \mathrm{~nm}$ by adding $\mathrm{CB}[8]$ into the solution containing Nap-PEG and $\mathrm{MV}$, corresponding to the typical CT band [36]. In addition, the solution color immediately turned into violet by mixing $\mathrm{CB}[8]$, Nap-PEG and MV (molar ratio=1:1:1), a direct proof for the generation of a CT complex (Fig. 1f). The association constants $\left(K_{\mathrm{a}}\right)$ of this ternary complex 
were determined by ITC, which provided the complexation thermodynamic behaviors and binding affinity. The $K_{1}$ value between $\mathrm{CB}[8]$ and $\mathrm{MV}$ was calculated to be $(1.53 \pm 0.05) \times 10^{6} \mathrm{M}^{-1}$ with a $1: 1$ complexation stoichiometry in PBS $(20.0 \mathrm{mM})$, verifying the strong interaction (Fig. 1g). By titration the solution of Nap-PEG into the solution of $\mathrm{CB}[8]$ and $\mathrm{MV}, K_{2}$ value was further calculated to be $(1.54 \pm 0.04) \times 10^{5} \mathrm{M}^{-1}$ (Fig. 1h), confirming the stepwise complexation. Thus, the final $K_{\mathrm{a}}$ value was about $2.36 \times 10^{11} \mathrm{M}^{-2}$, which demonstrated that the complexation was extremely stable in PBS. In order to assess the host-guest complexation in more complicated environment, the titration was conducted in cell culture medium containing fetal bovine serum, peptides and other substances, which possibly interfered the non-covalent interactions. As shown in Fig. 1i, the corresponding $K_{2}$ value was measured to be $(2.23 \pm 0.17) \times 10^{4} \mathrm{M}^{-1}$ in Dulbecco's Modified Eagle Medium (DMEM). It should be noted that the solubility of $\mathrm{CB}[8]$ was extremely poor in DMEM, thus the $K_{1}$ value could not be detected using ITC. By the formation of an inclusion complex with MV, the solubility of the formed complex was enough for ITC characterization. Compared with the $K_{2}$ values in PBS, the binding affinity showed an obvious decrease in cell culture medium, but still maintained a high level, which could guarantee the complexation. These studies revealed that the 1:1:1 ternary complexation between CB[8], NapPEG and MV was stable in physiological environment, such as PBS and culture medium.

\section{Fabrication of supramolecular nanomedicine and in vitro studies}

With the molecular recognition in hand, we constructed a supramolecular copolymer using CB[8], Nap-PEG and PCL-MV, in which the hydrophobic and hydrophilic segments were linked by $\mathrm{CB}[8]$. The amphiphilic nature of this supramolecular copolymer allowed to self-assemble into nanoparticles with a hydrophobic core to encapsulate a hydrophobic anticancer drug, like DOX. Indeed, a supramolecular nanomedicine (SNM@DOX) was successfully obtained with a drug loading content of $18.4 \%$. Transmission electron microscopy (TEM) was used to reveal the size and morphology of SNM@DOX. Figure 2a indicated that nanoparticulate assemblies were found in TEM image $90-120 \mathrm{~nm}$ in diameter. The average diameter of SNM@DOX was determined to be $164.2 \mathrm{~nm}$ by dynamic light scattering (DLS), which was a little bigger than the size obtained from TEM because of the swelling effect in aqueous solution (Fig. 2b). Before in vitro and in vivo studies, the stability of SNM@DOX was assessed
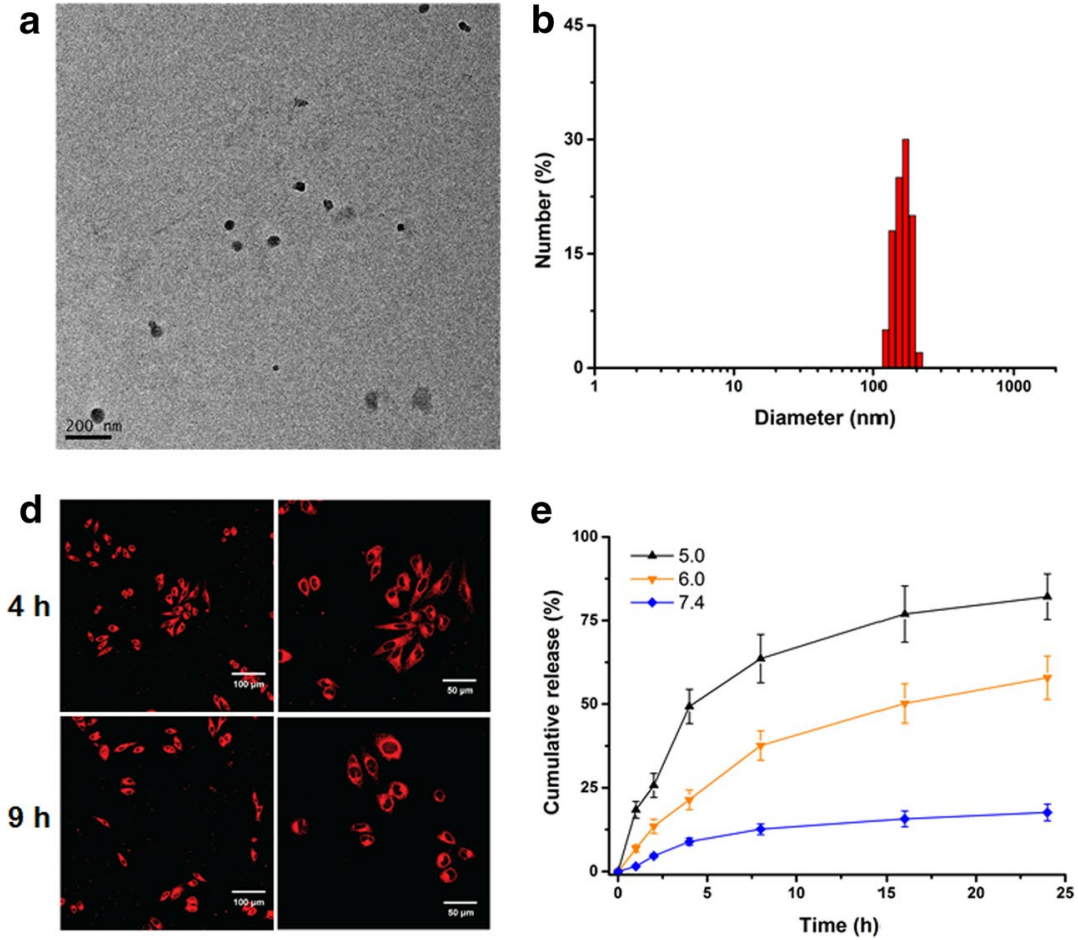
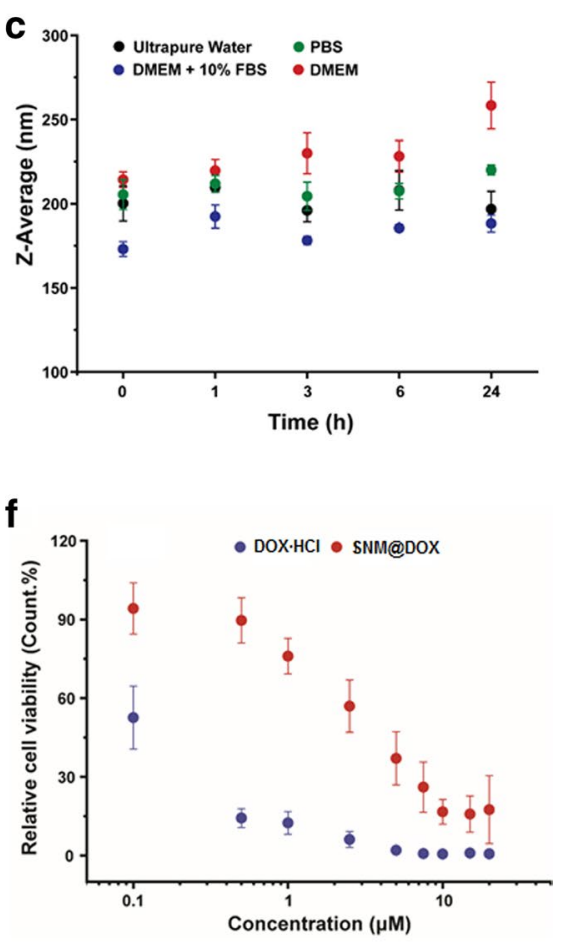

Fig. 2 a TEM image of SNM@DOX. $\mathbf{b}$ DLS result of SNM@DOX in PBS. $\mathbf{c}$ The average diameters of SNM@DOX in different solutions. $\mathbf{d}$ CLSM images of HepG2 cells cultured with SNM@DOX for 4 and $9 \mathrm{~h}$, respectively. The right images are the enlarged ones at the same time point. e Release profiles of DOX from SNM@DOX at different pH values. f Cytotoxicity of DOX.HCl and SNM@DOX against HepG2 cells 
by DLS measurements in different solutions, including water, PBS, DMEM and DMEM + FBS, respectively. Figure $2 \mathrm{c}$ showed that negligible changes in mean diameter were detected, suggesting that SNM@DOX maintained stable in these solutions attributing to the strong binding affinity of this host-guest molecular recognition, ensuring the biomedical applications of SNM@DOX.

Confocal laser scanning microscopy (CLSM) was utilized to reveal the cellular uptake of SNM@DOX (Fig. 2d), which showed bright red signal in cytoplasma after $4 \mathrm{~h}$ incubation. The intracellular fluorescence of DOX became more intensive and some signal appeared in the nucleus by extending the culture time to $9 \mathrm{~h}$. It should be emphasized that the endocytosis pathway of SNM@DOX was different from that of free DOX.HCl (Additional file 1: Fig. S17), which translocated into nucleus immediately after cellular internalization. DOX containing an amine group can be protonated in acidic environment, thus accelerating its release from the nanomedicine. To mimic the $\mathrm{pH}$ gradient from blood stream to the endo/lysosome, release behaviors of DOX from SNM@DOX were carried out at pH 7.4, 6.0, and 5.0, respectively (Fig. 2e). SNM@DOX kept stable in PBS at $\mathrm{pH}$ 7.4, only small amount (17.6\%) of DOX released from SNM@DOX within 24 h incubation. The release rate and amount speed up effectively at low $\mathrm{pH}$ value, $57.9 \%$ of DOX was released from SNM@DOX at pH 6.0 and 82.1\% at $\mathrm{pH} 5.0$ within the same period, respectively.

A 3-(4,5'-dimethylthiazol-2'-yl)-2,5-diphenyl tetrazolium bromide (MTT) assay was then chosen to evaluate the cytotoxicity of the delivery vehicles. Little influence on cell viability of $\mathrm{CB}[8]$, Nap-PEG and PCL-MV even at a relatively high concentration (Additional file 1: Fig. S18-20), an indicator of excellent biocompatibility. The anticancer efficacy of SNM@DOX against HepG2 cancer cells was assessed by CCK- 8 assay. The half maximal inhibitory concentration $\left(\mathrm{IC}_{50}\right)$ values of SNM@DOX were determined to be $10.7 \pm 1.14$ and $3.51 \pm 0.41 \mu \mathrm{M}$ after 24 and $48 \mathrm{~h}$ incubation, respectively (Fig. 2f, Additional file 1: Fig. S21). Compared with the free drug, the toxicity of DOX attenuated after nanoformulation mainly ascribing to the changes in endocytosis pathway and time-dependent release inside cells, while the cytotoxicity evaluation also elucidated that SNM@DOX kept satisfactory anticancer capability.

\section{In vivo anti-tumor performances}

The pharmacokinetic behaviors and time-dependent biodistributions of SNM@DOX were investigated before in vivo anti-tumor treatment, and free $\mathrm{DOX} \cdot \mathrm{HCl}$ was chosen as a control. After intravenous (i.v.) injection, the blood was collected at different time post injections. By plotting the drug amount in blood versus injection time, the circulation half-life could be obtained. DOX. $\mathrm{HCl}$ was cleared out from body rapidly with a half-life of $0.30 \pm 0.03 \mathrm{~h}$, while the circulation half-life of SNM@ DOX prolonged to $1.47 \pm 0.16 \mathrm{~h}$ after nanoformulation benefiting from the EPR effect (Fig. 3a). The area under the curve of SNM@DOX was also much larger than that of DOX $\cdot \mathrm{HCl}$, which provided the possibility of enhanced drug accumulation in the tumor. The preferred tumor accumulation of SNM@DOX was supported by timedependent biodistribution analysis. The intratumoral amount of SNM@DOX was $5.82 \pm 0.86 \% \mathrm{ID} / \mathrm{g}$ at $12 \mathrm{~h}$ post i.v. injection, which increased to $7.41 \pm 0.92 \% \mathrm{ID} / \mathrm{g}$ and $5.24 \pm 0.78 \% \mathrm{ID} / \mathrm{g}$ at $24 \mathrm{~h}$ and $48 \mathrm{~h}$ post injection, respectively (Fig. 3b). The "brushlike" PEG shell of SNM@DOX prevented protein adsorption that was favorable to prolong blood circulation time, facilitating the accumulation of SNM@DOX in tumor sites [36-40]. In contrast, the tumor accumulation of DOX. $\mathrm{HCl}$ was much lower, the highest amount in tumor was $2.33 \pm 0.42 \% \mathrm{ID} / \mathrm{g}$ occurred at $12 \mathrm{~h}$ post injection, and the drug eliminated from body quickly (Fig. 3b).

As a highly sensitive and noninvasive nuclear imaging technique, PET is widely used as a diagnostic molecular imaging tool by clinicians for preclinical and clinical imaging. By administrating a small amount of radiotracer, PET imaging can provide quantitative readout of pharmacokinetics, organ/tissue efficiency and tumor targeting efficiency in vivo, facilitating drug discovery and radiopharmaceutical development. A chelator Nap-DFO was obtained through three-step synthesis, which could chelate radioactive ${ }^{89} \mathrm{Zr}$ and further modified the supramolecular nanoparticles through host-guest complexation to afford ${ }^{89} \mathrm{Zr}$ SNM@DOX for PET imaging (Fig. 3c). The mice bearing HepG2 tumor were intravenously injected with ${ }^{89} \mathrm{Zr}$ SNM@DOX imaged at various time points. The dynamic biodistributions and accumulations in main organs could be clearly visualized in the wholebody PET images shown in Additional file 1: Fig. S23, such as liver and tumor. The prolonged circulation time of the supramolecular nanomedicine was also confirmed by PET imaging, in which intensive signal was observed in heart at $1 \mathrm{~h}$ post injection. Intriguingly, ${ }^{89} \mathrm{Zr}$ SNM@ DOX exhibited an efficient accumulation in tumor sites in a time-dependent manner after injection. Quantitative analysis in the region-of-interest of these images coincided with the results in Fig. 3d, the intra-tumoral amount of ${ }^{89} \mathrm{Zr}$ SNM@DOX was $0.92 \% \mathrm{ID} \mathrm{g}^{-1}$ at $1 \mathrm{~h}$ post injection, which increased to 4.14 and $6.52 \% \mathrm{ID} / \mathrm{g}$ at 7 and $24 \mathrm{~h}$ post injection, respectively.

In vivo anti-tumor ability of SNM@DOX was evaluated using HepG2 tumor-bearing mice that were intravenously injection with PBS, SNPs, free DOX. $\mathrm{HCl}$, and SNM@DOX, respectively. The tumors grew rapidly for 

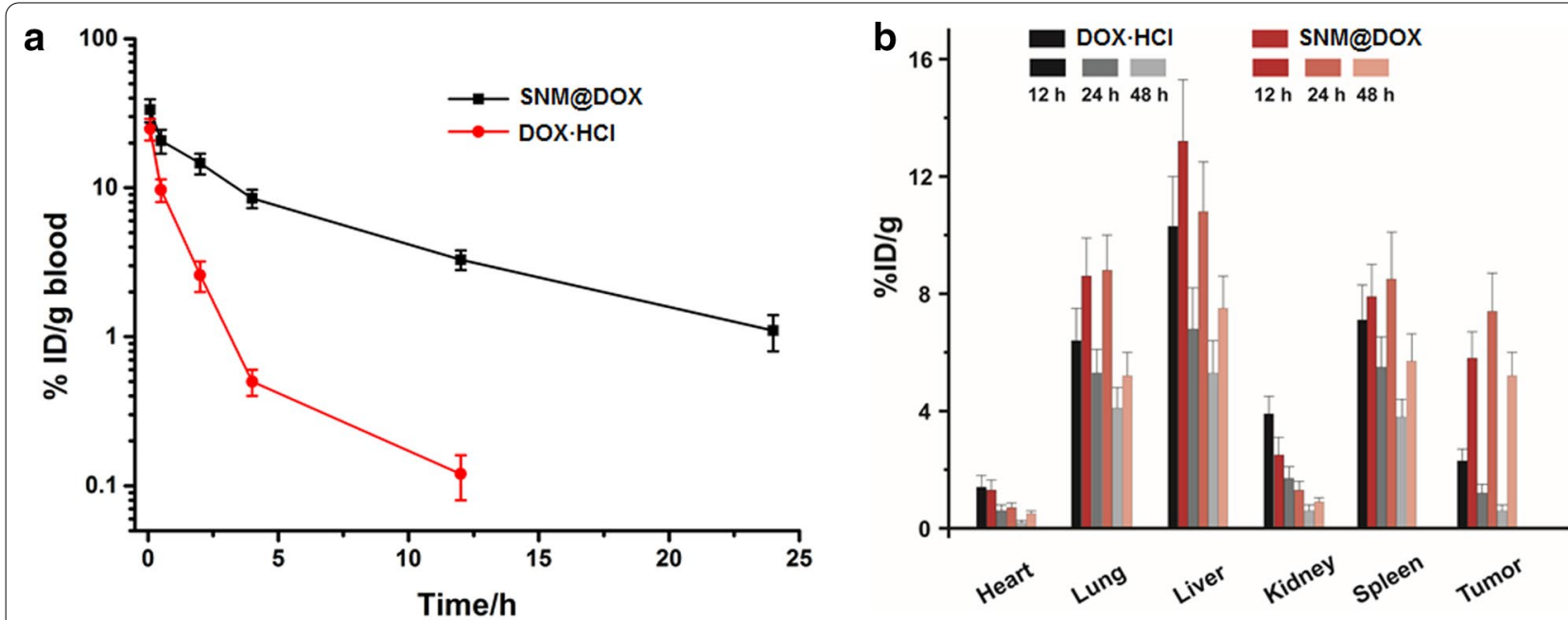

C

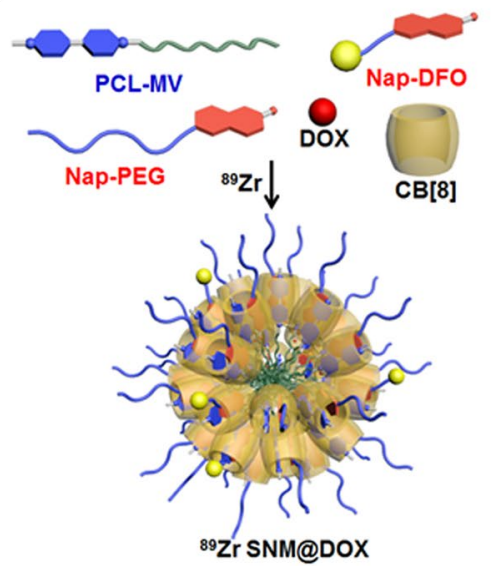

d

$1 \mathrm{~h}$

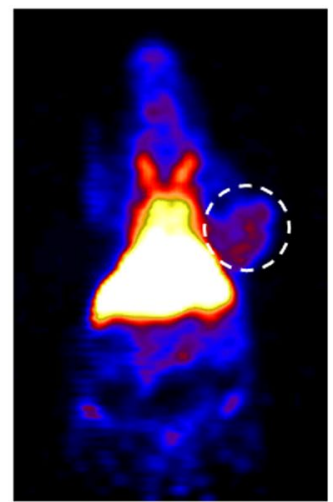

$7 \mathrm{~h}$

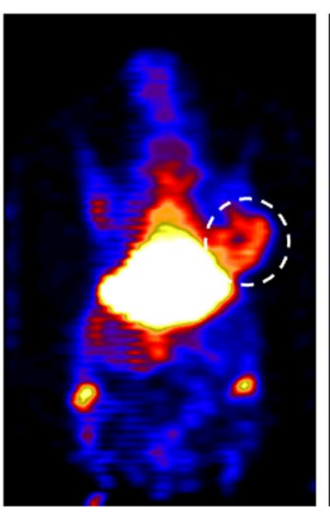

$24 \mathrm{~h}$

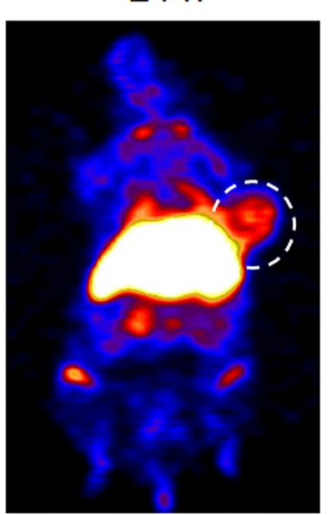

$10 \%$

Fig. 3 a Plasma concentration of DOX.HCl and SNM@DOX versus time after i.V. injection. b Time-dependent biodistributions of DOX.HCl and SNM@DOX in the main organs. c Schematic illustration of the preparation of ${ }^{89} \mathrm{Zr}$ SNM@DOX through multi-components self-assembly followed by radiolabelling. d PET images of the mice bearing HepG2 tumor at different time post i.v. injection of ${ }^{89} \mathrm{Zr}$ SNM@DOX

the mice treated with PBS or SNPs, the average tumor volume respectively increased to 912 and $1155 \mathrm{~mm}^{3}$ after 18 days (Fig. 4a), suggesting that the delivery vehicles had no influence on the tumor inhibition. Compared with the control mice treated with PBS, the formulation of free DOX. $\mathrm{HCl}$ resulted in a moderate tumor inhibition mainly attributing to the poor tumor accumulation and fast blood clearance, the inhibition rate was calculated to be $38.5 \%$ at the end day of therapy. In marked contrast, the tumor growth was effectively delayed for the mice administrated with SNM@DOX with a tumor inhibition rate of $62.6 \%$ (Fig. 4b), demonstrating the high antitumor efficacy of SNM@DOX treatment. The excellent therapeutic performance was further verified by hematoxylin and eosin (H\&E) staining, in which highest level of apoptotic and necrotic cancer cells were found characterized by nuclear shrinkage and fragmentation for the mice treated with SNM@DOX (Fig. 4c).
Body weight changes were carefully monitored during therapeutic period to assess the systemic toxicity. The body weight decreased obviously at the firstly ten days for the mice administrated with DOX. $\mathrm{HCl}$ because of its side effects (Additional file 1: Fig. S24). The systemic toxicity of the loaded drug greatly relieved by the formation of nanoformulation, negligible changes in body weight was observed during therapy for mice injected with SNM@DOX. The clinical use of DOX and other quinone-hydroquinone anticancer anthracyclines is limited by a dose-related cardiotoxicity, a leading factor for drug withdrawals. Compared with healthy mice, histological analysis of heart tissue indicated that no obvious tissue lesions were detected for the mice injected with SNM@ DOX (Additional file 1: Fig. S25). Moreover, the hepatotoxicity of the mice treated with DOX.HCl or SNM@ DOX was assessed by detecting the level of alkaline phosphatase (ALP), aspartate aminotransferase (AST), and 

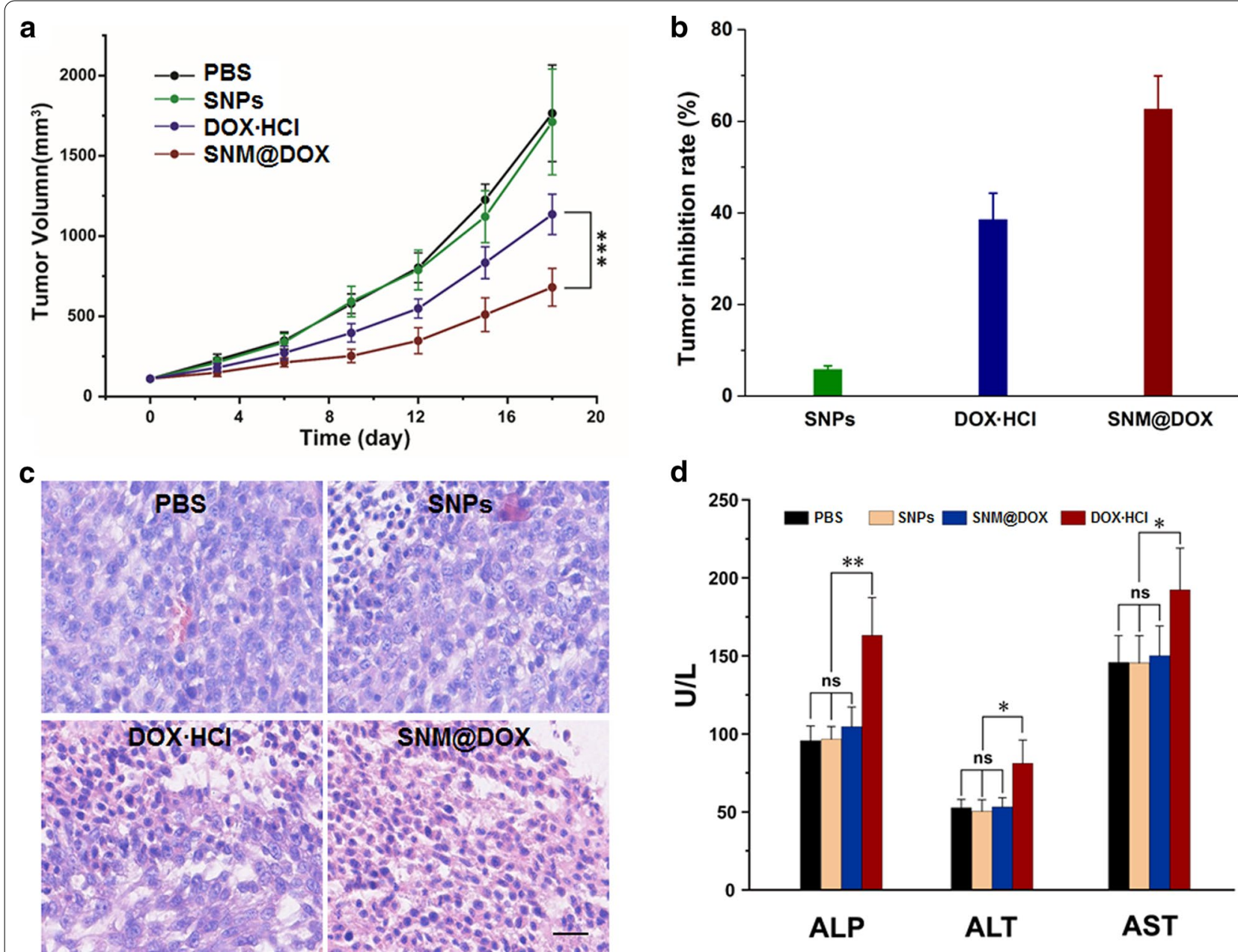

Fig. 4 a Tumor volume changes of the mice administrated with different formulations. ${ }^{* *} p<0.001$. b Tumor inhibition rate of the mice administrated with different formulations. $\mathbf{c}$ H\&E staining of the tumor tissues from the mice treated with PBS, SNPs, DOX.HCl, and SNM@DOX, respectively. Scale bar is $50 \mu \mathrm{m}$. $\mathbf{d}$ Blood biochemistry tests of ALP, AST, and ALT from the mice treated with different formulations. ${ }^{*} p<0.05$, ${ }^{* *} p<0.01$

alanine aminotransferase (ALT) in blood (Fig. 4d). An apparent elevation of these parameters was found for the mice receive free $\mathrm{DOX} \cdot \mathrm{HCl}$, while these indicators were normal for the mice injected with SNM@DOX when compared with the healthy mice. Benefiting from the EPR effect and high stability of SNM@DOX, undesirable adverse reactions of the anticancer drug were successfully attenuated through this supramolecular formulation.

\section{Conclusions}

In conclusion, the ternary host-guest complexation between $\mathrm{MV}$, napththyl derivative and $\mathrm{CB}[8]$ in physiological environment was fully investigated. ${ }^{1} \mathrm{H}$ NMR, ITC and UV-vis measurements collectively demonstrated that this ternary molecular recognition was stable in PBS and even cell culture medium containing FBS. The strong binding affinity in these aqueous solutions guaranteed the stability of supramolecular assemblies prepared from this recognition, which was extremely important for biomedical applications. A supramolecular diblock copolymer was constructed based on this 1:1:1 ternary complexation, which was further utilized to encapsulate a hydrophobic anticancer drug to afford a supramolecular nanomedicine SNM@DOX. Owing to the EPR effect, sophisticated engineering and non-covalent interactions, SNM@DOX possessed several advantages, such as high blood stability, prolonged circulation half-life, and abundant tumor accumulation. The delivery, biodistributions and accumulations of SNM@DOX in main organs were captured in real time through PET imaging by fully exploiting the host-guest complexation using a napththyl-modified chelator Nap-DFO. In vivo studies 
demonstrated the excellent therapeutic efficacy and attenuated systemic toxicity of SNM@DOX arising from the supramolecular nanoformulations. This pioneering work paves the way for the fabrication of supramolecular theranostics with promissing potentials in clinical trials on the basis of non-covalent interactions.

\section{Materials and methods}

1,5,7-triazabicyclo[4.4.0]dec-5-ene

(TBD),

$\varepsilon$-caprolactone, 6-bromo-1-hexanol, $p$-toluenesulfonyl chloride, methoxyl polyethylene glycol (mPEG-OH, $M_{\mathrm{w}}=2 \mathrm{kDa}$ ) were purchased from Sigma-Aldrich. Solvents were either employed as purchased or dried according to procedures described in the literature. Millipore ultrapure water was obtained on a Milli-Q purification system. Transmission electron microscopy (TEM) investigations were carried out on a HT-7700 instrument. UV-vis absorption spectra were recorded by using a Hitachi U-3010 spectrophotometer. Confocal laser scanning microscopy (CLSM) images was recorded on a LSM710META (Zeiss) microscope. Gel permeation chromatography (GPC) was conducted on a Waters Chromatography, Inc. (Milford, MA) system using THF containing $0.05 \mathrm{M} \mathrm{LiBr}$ as eluent. The sizes of the nanoformulations were determined by a DLS analyzer (Zetasizer Nano ZS90 Malvern Instruments, Malvern) with a detection angle of $90^{\circ}$ at $25^{\circ} \mathrm{C}$ using an incident $\mathrm{He}-\mathrm{Ne}$ laser $(\lambda=633 \mathrm{~nm})$. ITC experiments were carried out with a Microcal VP-ITC calorimeter at $298.1 \mathrm{~K}$. The anticancer efficacy was determined by using Cell Counting Kit-8 (CCK-8, Solarbio, Beijing) according to the instructions of the manufacturer. The absorbance of the bioreduced soluble formazan product was measured at $450 \mathrm{~nm}$ using a TECAN Infinite F200 PRO. H\&E tissue and cell staining was performed by $\mathrm{BBC}$ Biochemical (Mount Vernon, WA) and the images were collected using a BX41 bright field microscopy (Olympus).

\section{Synthesis of PCL-MV}

The synthetic routes of PCL-MV and Nap-PEG were illustrated in Additional file 1: Scheme S1. To a solution of 6-bromo-1-hexanol (181 $\mathrm{mg}, 1.00 \mathrm{mmol})$ and $\varepsilon$-caprolactone $(4.56 \mathrm{~g}, 40.0 \mathrm{mmol})$ in anhydrous $\mathrm{CH}_{2} \mathrm{Cl}_{2}$ $(10 \mathrm{~mL})$, TBD (139 mg, $1.00 \mathrm{mmol})$ was added and the mixture was stirred at room temperature. After $20 \mathrm{~min}$, the reaction was quenched by adding $1 \mathrm{~mL}$ of acetic acid. The resulting solution was precipitated into an excess of diethyl ether. After filtration, the sediments was dissolved in $\mathrm{CH}_{2} \mathrm{Cl}_{2}$ and precipitated into an excess of diethyl ether; the above dissolution-precipitation cycle was repeated three times. After drying in a vacuum oven overnight at room temperature, $\mathrm{PCL}-\mathrm{Br}$ was obtained as a white solid (4.45 g, yield: 93.9\%). The molecular weight and composition of PCL-Br were determined by ${ }^{1} \mathrm{H}$ NMR spectroscopy (Additional file 1: Fig. S1) and GPC (Additional file 1: Fig. S2).

A mixture of PCL-Br (1.15 g) and excessive MVI (700 mg) in DMF $(10 \mathrm{~mL})$ was stirred at $85{ }^{\circ} \mathrm{C}$ overnight. After reaction, the solvent was poured into $150 \mathrm{~mL}$ of $\mathrm{H}_{2} \mathrm{Cl}_{2}$, and the mixture was washed with water for three times. The solvent was evaporated and the residue was dissolved in $10 \mathrm{~mL}$ of THF. The resulting solution was precipitated into an excess of diethyl ether. The above dissolution-precipitation cycle was repeated three times. The solid was dried overnight in a vacuum to give a pale yellow powder with a yield of $92.7 \%$. The molecular weight and composition of PCL-MV were determined by ${ }^{1} \mathrm{H}$ NMR spectroscopy (Additional file 1: Fig. S3) and GPC (Additional file 1: Fig. S4).

\section{Synthesis of Nap-PEG}

mPEG-OH (10.0 g, $5.00 \mathrm{mmol})$ and $\mathrm{NaOH}(4.00 \mathrm{~g}$, $100 \mathrm{mmol}$ ) were dissolved in the mixture of THF and $\mathrm{H}_{2} \mathrm{O}\left(150 \mathrm{~mL}, \mathrm{THF} / \mathrm{H}_{2} \mathrm{O}=1 / 1, v / v\right)$. $p$-Toluenesulfonyl chloride $(5.70 \mathrm{~g}, 30.0 \mathrm{mmol})$ was dissolved in $30 \mathrm{ml}$ of THF, and the solution was droply added into the mPEG$\mathrm{OH}$ solution at $0{ }^{\circ} \mathrm{C}$. The mixture was further stirred at room temperature overnight. The organic solvent was evaporated, and the solution was extracted by $\mathrm{CH}_{2} \mathrm{Cl}_{2}$ $(3 \times 50 \mathrm{~mL})$. The organic phase was further washed with water for three times to eliminate the excessive $\mathrm{NaOH}$ and $p$-toluenesulfonyl chloride. The solvent was concentrated into $10 \mathrm{~mL}$, and the resulting solution was precipitated into an excess of diethyl ether. The above dissolution-precipitation cycle was repeated three times to afford mPEG-OTs without further purification.

A mixture containing mPEG-OTs $(2.15 \mathrm{~g})$, 6-methoxy-2-naphthol $(1.74 \mathrm{~g}, 10.0 \mathrm{mmol})$ and $\mathrm{K}_{2} \mathrm{CO}_{3}(2.76 \mathrm{~g}$, $20.0 \mathrm{mmol})$ in $\mathrm{CH}_{3} \mathrm{CN}(50 \mathrm{~mL})$ was added to a roundbottom flask under nitrogen atmosphere and heated at reflux for $12 \mathrm{~h}$. The organic phase was obtained after filtration and the solvent was removed by rotary evaporation to afford the crude product. The residue was dissolved in $5 \mathrm{~mL}$ of $\mathrm{CH}_{2} \mathrm{Cl}_{2}$, and the resulting solution was precipitated into an excess of diethyl ether. The above dissolution-precipitation cycle was repeated three times. The solid was dried overnight in a vacuum to give a dark green powder with a yield of $87.4 \%$. The molecular weight and composition of PCL-MV were determined by ${ }^{1} \mathrm{H}$ NMR spectroscopy (Additional file 1: Fig. S5) and GPC (Additional file 1: Fig. S6).

\section{Synthesis of Nap-DFO}

The synthetic route of Nap-DFO was illustrated in Additional file 1: Scheme S2. A mixture containing tert-butyl 
$\mathrm{N}$-(2-bromoethyl)carbamate (2.24 g, $10 \mathrm{mmol}), 6$-methoxy-2-naphthol $(0.87 \mathrm{~g}, 5.00 \mathrm{mmol})$ and $\mathrm{K}_{2} \mathrm{CO}_{3}(2.76 \mathrm{~g}$, $20.0 \mathrm{mmol})$ in $\mathrm{CH}_{3} \mathrm{CN}(50 \mathrm{~mL})$ was added to a roundbottom flask under nitrogen atmosphere and heated at reflux for $12 \mathrm{~h}$. The organic phase was obtained after filtration and the solvent was removed by rotary evaporation to afford the crude product, which was isolated by flash column chromatography to give Nap-Boc as a gray solid with a yield of $76.8 \% .{ }^{1} \mathrm{H}$ NMR (Additional file 1: Fig. S7), ${ }^{13} \mathrm{C}$ NMR (Additional file 1: Fig. S8) and mass (Additional file 1: Fig. S9) spectra were utilized to confirm the preparation of Nap-Boc.

Trifluoroacetic acid $(2.00 \mathrm{~mL})$ was added to the solution of Nap-Boc $(0.63 \mathrm{~g}, 2 \mathrm{mmol})$ in $\mathrm{CH}_{2} \mathrm{Cl}_{2}(15 \mathrm{~mL})$, and the mixture was stirred at room temperature for $8 \mathrm{~h}$. The solvent was removed by rotary evaporation and the compound was washed by methanol for three times to give Nap- $\mathrm{NH}_{2}$ as a brown solid with a yield of $64.5 \% .{ }^{1} \mathrm{H}$ NMR (Additional file 1: Fig. S10), ${ }^{13} \mathrm{C}$ NMR (Additional file 1: Fig. S11) and mass (Additional file 1: Fig. S12) spectra were utilized to confirm the preparation of Nap- $\mathrm{NH}_{2}$. Nap-DFO was obtained by labelling Nap- $\mathrm{NH}_{2}$ with NCSDFO, and the successful preparation of Nap-DFO was verified by ${ }^{1} \mathrm{H}$ NMR (Additional file 1: Fig. S13), ${ }^{13} \mathrm{C}$ NMR (Additional file 1: Fig. S14) and mass spectrum (Additional file 1: Fig. S15).

\section{Preparation of supramolecular nanomedicine}

DOX (8.00 mg), PCL-MV (18.5 mg) and Nap-PEG $(11.2 \mathrm{mg})$ were dissolved in DMSO $(10 \mathrm{~mL}), 10 \mathrm{~mL}$ of aqueous solution containing $\mathrm{CB}[8](1.00 \mathrm{mg} / \mathrm{mL})$ was droply added into the mixture solution. After stirring in the dark for $2 \mathrm{~h}$, the resulting mixture was sealed in dialysis bags with a molecular weight cut-off of $3.5 \mathrm{kDa}$ and dialyzed against DI water for $12 \mathrm{~h}$ to remove free DOX and $\mathrm{CB}[8]$. The drug loading content was estimated to be $18.4 \%$ by using UV spectroscopy. For the preparation of ${ }^{89} \mathrm{Zr}$ labelled nanomedicine, DOX (8.00 mg), PCL-MV (18.5 mg), Nap-DFO (0.300 mg), and Nap-PEG (10.5 mg) were dissolved in DMSO $(10 \mathrm{~mL}), 10 \mathrm{~mL}$ of aqueous solution containing $\mathrm{CB}[8](1.00 \mathrm{mg} / \mathrm{mL})$ was droply added into the mixture solution. After stirring in the dark for $2 \mathrm{~h}$, the resulting mixture was sealed in dialysis bags with a molecular weight cut-off of $3.5 \mathrm{kDa}$ and dialyzed against DI water for $12 \mathrm{~h}$ to remove Nap-DFO, free DOX and $\mathrm{CB}[8]$. SNM@DOX was labelled with ${ }^{89} \mathrm{Zr}$ by mixing the nanomedicine with radioactive isotope at $37{ }^{\circ} \mathrm{C}$ for $1 \mathrm{~h}$ under constant stirring.

\section{Drug release studies}

In vitro released profiles of DOX from SNM@DOX at different $\mathrm{pH}$ value were monitored using the dialysis method. The SNM@DOX was dissolved in $25 \mathrm{~mL}$ of distilled water and sealed in dialysis bags with a molecular weight cut-off of $2 \mathrm{kDa}$ at $\mathrm{pH} 7.4,6.0$, and 5.0, respectively. The dialysis apparatus was agitated on an orbital shaker at $100 \mathrm{rpm}$ at $37^{\circ} \mathrm{C}$. At designated time intervals, $1 \mathrm{~mL}$ of medium was taken out from the $25 \mathrm{~mL}$ solution out of the dialysis bag for UV detection and was then put back to the original system. The DOX concentration was calculated with a standard curve calibrated with DOX samples of known concentrations.

\section{Cell cultures}

HepG2 cells were cultured in Dulbecco's modified Eagle's medium (DMEM) containing 10\% fetal bovine serum (FBS) and 1\% penicillin/streptomycin. Cells grew as a monolayer and were detached upon confluence using trypsin $(0.5 \% w / v$ in PBS). The cells were harvested from the cell culture medium by incubating in a trypsin solution for $5 \mathrm{~min}$. The cells were centrifuged, and the supernatant was discarded. A $3 \mathrm{~mL}$ portion of serumsupplemented DMEM was added to neutralize any residual trypsin. The cells were resuspended in serumsupplemented DMEM at a concentration of $1 \times 10^{4}$ cells/ $\mathrm{mL}$. Cells were cultured at $37^{\circ} \mathrm{C}$ and $5 \% \mathrm{CO}_{2}$.

\section{Evaluation of cytotoxicity}

The cytotoxicities of CB[8], PCL-MV, Nap-PEG, DOX.HCl, and SNM@DOX against HepG2 cells were determined by MTT or CCK-8 assay in a 96-well cell culture plate. All solutions were sterilized by filtration with a $0.22 \mu \mathrm{m}$ filter before tests. HepG2 cells were seeded at a density of $1 \times 10^{4}$ cells/well in a 96-well plate, and incubated for $24 \mathrm{~h}$ for attachment. Cells were then incubated with CB[8], PCL-MV, Nap-PEG, DOX.HCl, and SNM@ DOX at various concentrations for $24 \mathrm{~h}$. After washing the cells with PBS buffer, $20 \mu \mathrm{L}$ of a MTT solution $(5 \mathrm{mg} /$ $\mathrm{mL}$ ) was added to each well. After $4 \mathrm{~h}$ of incubation at $37{ }^{\circ} \mathrm{C}$, the MTT solution was removed, and the insoluble formazan crystals that formed were dissolved in 100 $\mu \mathrm{L}$ of dimethylsulfoxide (DMSO). The absorbance of the formazan product was measured at $570 \mathrm{~nm}$ using a spectrophotometer (Bio-Rad Model 680). For CCK-8 assay, WST-8 [2-(2-methoxy-4-nitrophenyl)-3-(4-nitrophenyl)-5-(2, 4-disulfophenyl)-2H-tetrazolium, monosodium salt] solution was added and cells were incubated for $4 \mathrm{~h}$ at $37{ }^{\circ} \mathrm{C}$ under $5 \% \mathrm{CO} 2$. The absorbance of each well was measured with a luminescence microplate reader (BioRad 680) at $450 \mathrm{~nm}$. Untreated cells in media were used as a control. All experiments were carried out with five replicates.

\section{Cellular internalization studies by CLSM}

HepG2 cells were treated with SNM@DOX (the concentration of DOX was $2.00 \mu \mathrm{M}$ ) in the culture medium at 
$37^{\circ} \mathrm{C}$ for 4 and $9 \mathrm{~h}$, respectively. The cells were washed three times with PBS and fixed with fresh $4.0 \%$ formaldehyde at room temperature for $15 \mathrm{~min}$. After washing with PBS, the cells were stained with DAPI $(1 \mu \mathrm{g} / \mathrm{mL})$ for $15 \mathrm{~min}$. The images were taken using a LSM-510 confocal laser scanning microscope (Zeiss, Germany) $(100 \times$ oil objective, $405 / 488 \mathrm{~nm}$ excitation).

\section{Animals and tumor models}

Female nude mice ( 4 weeks old, $\sim 20$ g body weight) were purchased from Zhejiang Academy of Medical Sciences and maintained in a pathogen-free environment under controlled temperature $\left(24{ }^{\circ} \mathrm{C}\right)$. Animal care and handling procedures were in agreement with the guidelines evaluated and approved by the ethics committee of Zhejiang University of Technology. Study protocols involving animals were approved by the Zhejiang University of Technology Animal Care and Use Committee. The female nude mice were injected subcutaneously in the right flank region with $200 \mu \mathrm{L}$ of cell suspension containing $2 \times 10^{6}$ HepG2 cells. The tumors were allowed to grow to $\sim 100 \mathrm{~mm}^{3}$ before experimentation. The tumor volume was calculated as (tumor length) $\times(\text { tumor width })^{2} / 2$. Relative tumor volumes were calculated as $V / V_{0}\left(V_{0}\right.$ was the tumor volume when the treatment was initiated).

\section{Pharmacokinetics and biodistribution}

For pharmacokinetic studies, the mice were randomly divided into two groups $(n=3)$. The aqueous solutions of free DOX.HCl, and SNM@DOX were i.v. injected via tail vein at a dose of $10.0 \mathrm{mg}$ DOX/kg. The blood samples $(0.1 \mathrm{~mL})$ were taken from the eye socket at the different time points post injection. The plasma was obtained by centrifugation at $3000 \mathrm{rpm}$ for $15 \mathrm{~min}$, and the amount of DOX in the plasma was assayed by HPLC. The DOX concentrations in the tumor tissues and organs were analyzed by HPLC. $200 \mu \mathrm{L}$ of $10 \%(w / v)$ tissue homogenate was added with $100 \mu \mathrm{L}$ of PBS. The above mixture was subsequently extracted with chloroform/isopropanol $(4: 1, v / v)$ by vortex mixing for $3 \mathrm{~min}$. After centrifugation at 10,000 rpm for $5 \mathrm{~min}$, the organic phase was separated and evaporated to dryness under a stream of nitrogen. The residue was dissolved in $200 \mu \mathrm{L}$ of mobile phase (methanol/water/acetic acid $=65: 35: 2, v / v / v)$. After centrifugation at 10,000 rpm for $5 \mathrm{~min}$, the supernatant was collected for HPLC analysis. The mice bearing HepG2 tumors were i.v. injected ${ }^{89} \mathrm{Zr}$ SNM@DOX and anesthetized with isoflurane $(1.0 \sim 2.0 \%)$ in oxygen delivered at a flow rate of $1.0 \mathrm{~L} / \mathrm{min}$. All PET imaging scans were conducted on a micro-PET/CT at different time post injection.

\section{In vivo anti-tumor evaluation}

The mice were divided into three treatment groups randomly $(n=5)$, when the mean tumor volume reached about $100 \mathrm{~mm}^{3}$ and this day was set as day 0 . Mice were administered intravenously with PBS, SNPs, free $\mathrm{DOX} \cdot \mathrm{HCl}(5.00 \mathrm{mg} \mathrm{DOX} / \mathrm{kg})$, and SNM@DOX (5.00 mg $\mathrm{DOX} / \mathrm{kg}$ ), respectively every 3 days for four times. Tumor volume and body weight were measured every 3 days. The tumor inhibition study was stopped on the 18th day. In the histological assay, the tissues were fixed in $4 \%$ paraformaldehyde for $24 \mathrm{~h}$. The specimens were dehydrated in graded ethanol, embedded in paraffin, and cut into $5 \mathrm{~mm}$ thick sections. The fixed sections were deparaffinized and hydrated according to a standard protocol and stained with hematoxylin and eosin (H\&E) for microscopic observation.

\section{Statistical analysis}

Data are presented as the mean \pm standard deviation. Statistical analysis of data was performed with one-way analysis of variance. The level of significance was defined at $" p<0.05, * * p<0.01,{ }^{* * * *} p<0.001$.

\section{Supplementary material}

Experimental details and supporting data. This material is available free of charge via the Internet at https://jnano biotechnology.biomedcentral.com/.

\section{Abbreviations}

CB[8]: Cucurbit[8] uril; ITC: Isothermal titration calorimetry; DOX: Doxrubincin; PEG: Polyethylene glycol; PCL: Poly( $\varepsilon$-caprolactone); EPR: Enhanced permeability and retention; PBS: Phosphate bufer saline; CT: Charge-transfer; $K_{a}$ : Association constants; TEM: Transmission electron microscopy; \%ID/g: Injected dose per gram; DLS: Dynamic light scattering; CLSM: Confocal laser scanning microscopy; MTT: 3-(4',5'-Dimethylthiazol-2'-yl)-2,5-diphenyl tetrazolium bromide; $I C_{50}$ : Half maximal inhibitory concentration; PET: Positron emission tomography; H\&E: Hematoxylin and eosin; ALT: Alanine amino transferase; AST: Aspartate aminotransferase; ALP: Alkaline phosphatase.

\section{Supplementary Information}

The online version contains supplementary material available at https://doi. org/10.1186/s12951-021-01076-z.

\footnotetext{
Additional file 1: Scheme S1. Synthetic routes to PCL-MV and Nap-PEG. Scheme S2. Synthetic routes to Nap-DFO. Fig. S1. ${ }^{1} \mathrm{H}$ NMR spectrum (400 $\mathrm{MHz}, \mathrm{CDCl} 3$, room temperature) of $\mathrm{PCL}-\mathrm{Br}$. Fig. S2. GPC curve of PCL-Br. Fig. S3. ${ }^{1} \mathrm{H}$ NMR spectrum ( $400 \mathrm{MHz}, \mathrm{CDCl} 3$, room temperature) of PCLMV. Fig. S4. GPC curve of PCL-MV. Fig. S5. ${ }^{1} \mathrm{H}$ NMR spectrum $(400 \mathrm{MHz}$, CDCl3, room temperature) of Nap-PEG. Fig. S6. GPC curve of Nap-PEG. Fig. S7. ${ }^{1} \mathrm{H}$ NMR spectrum ( $400 \mathrm{MHz}, \mathrm{CDCl} 3$, room temperature) of NapBoc. Fig. S8. 13C NMR spectrum (100 MHz, CDCl3, room temperature) of Nap-Boc. Fig. S9. ESI IT-TOF results of Nap-Boc. $[\mathrm{M}+\mathrm{Na}]+=340.1494$. Fig. S10. ${ }^{1} \mathrm{H}$ NMR spectrum (400 MHz, DMSO-d6, room temperature) of Nap-NH2. Fig. S11. ${ }^{13} \mathrm{C}$ NMR spectrum (100 MHz, DMSO-d6, room temperature) of Nap-NH2. Fig. S12. ESI IT-TOF results of Nap-NH2. [M + $\mathrm{H}]+=218.1142$. Fig. S13. ${ }^{1} \mathrm{H}$ NMR spectrum $(400 \mathrm{MHz}$, DMSO-d6, room temperature) of Nap-DFO. Fig. S14. ${ }^{13} \mathrm{C}$ NMR spectrum (100 MHz, DMSOd6, room temperature) of Nap-DFO. Fig. S15. ESI IT-TOF result of Nap-DFO.
} 
$[\mathrm{M}-\mathrm{H}]-=775.3225$. Fig. S16. ${ }^{1} \mathrm{H}$ NMR spectra of a Nap-PEG, b Nap-PEG $+\mathrm{CB}[8]$ and $\mathrm{C}$ CB[8] in D2O. Fig. S17. CLSM images of HepG2 cells cultured with DOX.HCl for $4 \mathrm{~h}$ and $9 \mathrm{~h}$, respectively. The right images are the enlarged ones at the same time point. Fig. S18. Cytotoxicity evaluation of CB[8] against HepG2 cells using an MTT assay. Fig. S19. Cytotoxicity evaluation of PCL-MV against HepG2 cells using an MTT assay. Fig. S20 Cytotoxicity evaluation of Nap-PEG against HepG2 cells using an MTT assay. Fig. S21. Cytotoxicity evaluation of DOX.HCl and SNM@DOX against HepG2 cells after $24 \mathrm{~h}$ incubation using a CCK-8 assay. Fig. S22. Release profiles of Nap-DFO from the nanoformulation in water or PBS. Fig. $\mathbf{S 2 3}$. Time-dependent biodistribution of 89Zr SNM@DOX in liver and tumor. Fig. S24. Body weight changes of the mice treated with different formulations. Fig. S25. H\&E staining of the heart tissues from a healthy mouse and $b$ the mouse treated with SNM@DOX at day 18.

\section{Acknowledgements}

This work was supported by the Tsinghua University Spring Breeze Fund (2021Z99CFZ007), the Starry Night Science Fund of Zhejiang University Shanghai Institute for Advanced Study (SN-ZJU-SIAS-006), the startup funding by Tsinghua University, the Natural Key Research and Development Program of China (2016YFC0103804), National Natural Science Foundation of China (81871379), and the Fundamental Research Funds for the Central Universities of China.

\section{Authors' contributions}

HW, ZC, FK and GY conceived and designed the method. HW, SQ, BB, JY, and DW performed the experiments. HW, JS and FK analyzed the data. HW and GY wrote the manuscript. All authors read and approved the final manuscript.

\section{Availability of data and material}

The datasets generated and/or analyzed during the current study are available from the corresponding authors on reasonable request.

\section{Declarations}

\section{Ethics approval and consent to participate}

All procedures were performed in accordance with the Regulations of the People's Republic of China on the Management of Laboratory Animals and approved by the ethics committee of Zhejiang University of Technology, P. R. China.

\section{Consent to participate \\ Not applicable.}

\section{Consent for publication}

Not applicable.

\section{Competing interest}

The authors declare no competing interests.

\section{Author details}

${ }^{1}$ Key Laboratory of Organic Optoelectronics and Molecular Engineering, Department of Chemistry, Tsinghua University, Beijing 100084, People's Republic of China. ${ }^{2}$ Department of Rehabilitation Medicine, The First Affiliated Hospital, College of Medicine, Zhejiang University, Hangzhou 310003, People's Republic of China. ${ }^{3}$ Department of Nuclear Medicine, Xijing Hospital, Fourth Military Medical University, Xi'an 400030, People's Republic of China. ${ }^{4}$ College of Materials Science and Engineering, Zhejiang University of Technology, Hangzhou 310014, People's Republic of China. ${ }^{5}$ Department of Pharmacy, School of Medicine, Zhejiang University City College, Hangzhou 310015, People's Republic of China.

Received: 21 August 2021 Accepted: 9 October 2021 Published online: 20 October 2021

\section{References}

1. Zhou J, Rao L, Yu G, Cook TR, Chen X, Huang F. Supramolecular cancer nanotheranostics. Chem Soc Rev. 2021;50:2839-91.

2. Webber MJ, Langer R. Drug delivery by supramolecular design. Chem Soc Rev. 2017:46:6600-20.

3. Yu G, Yang Z, Fu X, Yung BC, Yang J, Mao Z, Shao L, et al. Polyrotaxanebased supramolecular theranostics. Nat Commun. 2018;9:766.

4. Feng Z, Zhang T, Wang H, Xu B. Supramolecular catalysis and dynamic assemblies for medicine. Chem Soc Rev. 2017;46:6470-9.

5. Zhou J, Yu G, Huang F. Supramolecular chemotherapy based on hostguest molecular recognition: a novel strategy in the battle against cancer with a bright future. Chem Soc Rev. 2017;46:7021-53.

6. Goor OJGM, Hendrikse SIS, Dankers PYW, Meijer EW. From supramolecular polymers to multi-component biomaterials. Chem Soc Rev. 2017;46:6621-37.

7. Cabral H, Nishiyama N, Kataoka K. Supramolecular nanodevices: from design validation to theranostic nanomedicine. Acc Chem Res. 2011:44(10):999-1008.

8. Zhu H, Wang H, Shi B, Shangguan L, Tong W, Yu G, et al. Supramolecular peptide constructed by molecular Lego allowing programmable selfassembly for photodynamic therapy. Nat Commun. 2019;10:2412.

9. Sato K, Hendricks MP, Palmer LC, Stupp SI. Peptide supramolecular materials for therapeutics. Chem Soc Rev. 2018;47:7539-51.

10. Yang K, Yu G, Yang Z, Yue L, Zhang X, Sun C, et al. Supramolecular polymerization-induced nanoassemblies for self-augmented cascade chemotherapy and chemodynamic therapy of tumor. Angew Chem Int Ed. 2021;60(32):17570-8.

11. Zhu H, Shangguan L, Shi B, Yu G, Huang F. Recent progress in macrocyclic amphiphiles and macrocyclic host-based supra-amphiphiles. Mater Chem Front. 2018;2:2152-74.

12. Wang $C$, Sang H, Wang $Y$, Zhu F, Hu X, Wang $X$, et al. Foe to friend: supramolecular nanomedicines consisting of natural polyphenols and bortezomib. Nano Lett. 2018;18(11):7045-51.

13. Gao J, Li J, Geng WC, Chen FY, Duan X, Zheng Z, et al. Biomarker displacement activation: a general host-guest strategy for targeted phototheranostics in vivo. J Am Chem Soc. 2018;140(14):4945-53.

14. Yu G, Chen X. Host-guest chemistry in supramolecular theranostics. Theranostics. 2019;9(11):3041-74.

15. Geng WC, Sessler JL, Guo DS. Supramolecular prodrugs based on hostguest interactions. Chem Soc Rev. 2020;49:2303-15.

16. Sun C, Wang Z, Yue L, Huang Q, Cheng Q, Wang R. Supramolecular induction of mitochondrial aggregation and fusion. J Am Chem Soc. 2020;142(39):16523-7.

17. Zhang Z, Yue YX, Xu L, Wang Y, Geng WC, Li JJ, et al. Macrocyclic-amphiphile-based self-assembled nanoparticles for ratiometric delivery of therapeutic combinations to tumors. Adv Mater. 2021;33(12):2007719.

18. Yu G, Zhao X, Zhou J, Mao Z, Huang X, Wang Z, et al. Supramolecular polymer-based nanomedicine: high therapeutic performance and negligible long-term immunotoxicity. J Am Chem Soc. 2018;140(25):8005-19.

19. Wang L, Li LL, Fan YS, Wang H. Host-guest supramolecular nanosystems for cancer diagnostics and therapeutics. Adv Mater. 2013;25(28):3888-98.

20. Xu Z, Jia S, Wang W, Yuan Z, Ravoo BJ, Guo DS. Heteromultivalent peptide recognition by co-assembly of cyclodextrin and calixarene amphiphiles enables inhibition of amyloid fibrillation. Nat Chem. 2019;11:86-93.

21. Wankar J, Kotla NG, Gera S, Rasala S, Pandit A, Rochev YA. Recent advances in host-guest self-assembled cyclodextrin carriers: implications for responsive drug delivery and biomedical engineering. Adv Funct Mater. 2020;30(44):1909049.

22. Pan YC, Hu XY, Guo DS. Biomedical applications of calixarenes: state of the art and perspectives. Angew Chem Int Ed. 2021;60(6):2768-94.

23. Wu D, Li Y, Yang J, Shen J, Zhou J, Hu Q, et al. Supramolecular nanomedicine constructed from cucurbit[8]uril-based amphiphilic brush copolymer for cancer therapy. ACS Appl Mater Interfaces. 2017;9(51):44392-401.

24. Gao C, Cheng Q, Li J, Chen J, Wang Q, Wei J, et al. Supramolecular macrophage-liposome marriage for cell-hitchhiking delivery and immunotherapy of acute pneumonia and melanoma. Adv Funct Mater. 2021. https://doi.org/10.1002/adfm.202102440.

25. Huang Q, Cheng Q, Zhang X, Yin H, Wang LH, Wang R. Alleviation of polycation-induced blood coagulation by the formation of polypseudorotaxanes with macrocyclic cucurbit[7]uril. ACS Appl Bio Mater. 2018;1(3):544-8. 
26. Chen K, Xu X, Guo J, Zhang X, Han S, Wang R, et al. Enhanced intracellular delivery and tissue retention of nanoparticles by mussel-inspired surface chemistry. Biomacromol. 2015;16(11):3574-83.

27. Lee DW, Park KM, Banerjee M, Ha SH, Lee T, Suh K, et al. Supramolecular fishing for plasma membrane proteins using an ultrastable synthetic host-guest binding pair. Nat Chem. 2011;3:154-9.

28. Samanta SK, Moncelet D, Briken V, Isaacs L. Metal-organic polyhedron capped with cucurbit[8] uril delivers doxorubicin to cancer cells. J Am Chem Soc. 2016:138(43):14488-96.

29. Tang B, Li W, Chang Y, Yuan B, Wu Y, Zhang M, et al. A supramolecular radical dimer: high-efficiency NIR-Il photothermal conversion and therapy. Angew Chem Int Ed. 2019;58(43):15526-31.

30. Zhang Y, Liu J, Yu Q, Wen X, Liu Y. Targeted polypeptide-microtubule aggregation with cucurbit[8]uril for enhanced cell apoptosis. Angew Chem Int Ed. 2019;58(31):10553-7.

31. Ruz P, Banerjee S, Khurana R, Barooah N, Sudarsan V, Bhasikuttan AC, et al. Metal-free supramolecular catalytic hydrolysis of ammonia borane through cucurbituril nanocavitands. ACS Appl Mater Interfaces. 2021;13(14):16218-26

32. Khurana R, Mohanty J, Barooah N, Bhasikuttan AC. Photoinduced emissive naphthalenediimide radical anion in the confinement of cucurbituril nanocavity in situ generation of gold nanoparticles. J Mol Liq. 2021;334:116023.

33. Barooah N, Kunwar A, Khurana R, Bhasikuttan AC, Mohanty J. Stimuliresponsive cucurbit[7]uril-mediated bsa nanoassembly for uptake and release of doxorubicin. Chem Asian J. 2017;12(1):122-9.

34. Yu G, Jie K, Huang F. Supramolecular amphiphiles based on host-guest molecular recognition motifs. Chem Rev. 2015;115(15):7240-303.
35. Barrow SJ, Kasera S, Rowland MJ, del Barrio J Scherman OA. Cucurbiturilbased molecular recognition. Chem Rev. 2015;115(22):12320-406.

36. Liu Y, Yu Y, Gao J, Wang Z, Zhang X. Water-soluble supramolecular polymerization driven by multiple host-stabilized charge-transfer interactions. Angew Chem Int Ed. 2010;49(37):6576-9.

37. Jiao Y, Xu JF, Wang Z, Zhang X. Visible-light photoinduced electron transfer promoted by cucurbit[8] uril-enhanced charge transfer interaction: toward improved activity of photocatalysis. ACS Appl Mater Interfaces. 2017;9(27):22635-40

38. Yu GC, Zhu BY, Shao L, Zhou J, Saha ML, Shi BB, et al. Host-guest complexation-mediated codelivery of anticancer drug and photosensitizer for cancer photochemotherapy. Proc Natl Acad Sci USA. 2019;116:6618-23.

39. Purushothaman B, Lee J, Hong S, Song JM. Multifunctional TPP-PEG-biotin self-assembled nanoparticle drug delivery-based combination therapeutic approach for co-targeting of GRP78 and lysosome. J Nanobiotechnol. 2020;18:102.

40. Xu Q, Ensign LM, Boylan NJ, Schön A, Gong X, Yang JC, et al. Impact of surface polyethylene glycol (PEG) density on biodegradable nanoparticle transport in mucus ex vivo and distribution in vivo. ACS Nano. 2015;9(9):9217-27.

\section{Publisher's Note}

Springer Nature remains neutral with regard to jurisdictional claims in published maps and institutional affiliations.
Ready to submit your research? Choose BMC and benefit from:

- fast, convenient online submission

- thorough peer review by experienced researchers in your field

- rapid publication on acceptance

- support for research data, including large and complex data types

- gold Open Access which fosters wider collaboration and increased citations

- maximum visibility for your research: over $100 \mathrm{M}$ website views per year

At BMC, research is always in progress.

Learn more biomedcentral.com/submissions 